\title{
Effect of AST on age-associated changes of vocal folds in a rat model.
}

\section{AUTHOR(S):}

Mizuta, Masanobu; Hirano, Shigeru; Hiwatashi, Nao; Kobayashi, Toshiki; Tateya, Ichiro; Kanemaru, Shin-Ichi; Nakamura, Tatsuo; Ito, Juichi

\section{CITATION:}

Mizuta, Masanobu ... [et al]. Effect of AST on age-associated changes of vocal folds in a rat model.. The Laryngoscope 2014, 124(10): E411-E417

\section{ISSUE DATE:}

2014-05-27

URL:

http://hdl.handle.net/2433/200222

\section{RIGHT:}

This is the peer reviewed version of the following article: Mizuta, M., Hirano, S., Hiwatashi, N., Kobayashi, T., Tateya, I. Kanemaru, S.-i., Nakamura, T. and Ito, J. (2014), Effect of AST on age-associated changes of vocal folds in a rat model. The Laryngoscope, 124: E411-E417, which has been published in final form at http://dx.doi.org/10.1002/lary.24733. This article may be used for non-commercial purposes in accordance with Wiley Terms and Conditions for Self-Archiving.; This is not the published version. Please cite only the published version.; この論文は出版社版でありません。引用の際 には出版社版をご確認ご利用ください。 
Effect of astaxanthin on age-associated changes of vocal folds in a rat model

(Abbreviation: Astaxanthin for vocal fold aging)

\author{
Masanobu Mizutaํㅗ MD \\ Shigeru Hirano ${ }^{1}, \mathrm{MD}, \mathrm{PhD}$ \\ Nao Hiwatashi ${ }^{1}, \mathrm{MD}$ \\ Toshiki Kobayashi, MD \\ Ichiro Tateya ${ }^{1}, \mathrm{MD}, \mathrm{PhD}$ \\ Shin-ichi Kanemaru2, 3, 1, MD, PhD \\ Tatsuo Nakamura ${ }^{4}, \mathrm{MD}, \mathrm{PhD}$ \\ Juichi Ito ${ }^{1}, \mathrm{MD}, \mathrm{PhD}$
}

${ }^{1}$ Department of Otolaryngology-Head and Neck Surgery, Graduate School of Medicine, Kyoto University, Kyoto.

${ }^{2}$ Department of Otolaryngology, Regenerative treatment for tympanic membrane, the Foundation for Biomedical Research and Innovation, Kobe.

${ }^{3}$ Department of Otolaryngology-Head and Neck Surgery, Kitano Hospital, Tazuke Kofukai Medical Research Institute, Osaka.

${ }^{4}$ Department of Bioartificial Organs, Institute for Frontier Medical Science, Kyoto University, Kyoto.

$\checkmark \quad$ This study was supported by the Advanced Research for Medical Products Mining Program of the National Institute of Biomedical Innovation (NIBIO).

$\checkmark$ To be presented at the 134th American Laryngological Association, Las Vegas, Nevada, U.S.A., May 14-15, 2014. 
$\checkmark$ Conflict of Interest: None

\section{Correspondence to:}

Shigeru Hirano, MD, PhD, Department of Otolaryngology-Head \& Neck Surgery, Graduate School of Medicine, Kyoto University, Sakyo-ku, Kyoto 606-8507, Japan.

Tel: +81-75-751-3346, Fax: +81-75-751-7225, E-mail: hirano@ent.kuhp.kyoto-u.ac.jp 


\section{Abstract}

Objectives: Reactive oxygen species (ROS) are associated with aging. Astaxanthin is a strong antioxidant and has been reported to prevent various ROS-induced diseases. In the current study, we investigated the effect of astaxanthin on age-associated histological and mRNA changes of vocal folds.

Study design: Prospective animal experiment with control.

Methods: Six-month-old Sprague-Dawley rats were fed on a normal powder diet with $0.01 \%(\mathrm{w} / \mathrm{w})$ astaxanthin (aged Ast-treated group) or without astaxanthin (aged sham-treated group). After 12 months of feeding, the larynges were harvested for histology, immunohistochemical detection of 4-hydroxy-2-nonenal (4-HNE) and quantitative real-time PCR for basic fibroblast growth factor (bFGF) and hepatocyte growth factor (HGF). Thirteen-week-old rats were used as a young control group (young group).

Results: The expression of 4-HNE, an oxidative stress marker, significantly increased in the two aged groups compared to the young group. Histological examination showed that the deposition of hyaluronic acid in the lamina propria was significantly reduced in the aged sham-treated group compared to the young group, but no significant difference was observed between the aged Ast-treated group and the young group. There were no significant differences in the mRNA expression of bFGF and HGF between the aged Ast-treated group and the young group, although the expression of these genes was significantly reduced in the aged sham-treated group as compared to the young group. Conclusions: These results suggest that astaxanthin has the potential to attenuate age-associated changes of vocal folds.

Key Words: astaxanthin, age-associated changes, vocal folds, reactive oxygen species 
Level of Evidence: N/A 


\section{Introduction}

Many hypotheses of aging have been proposed including the reactive oxygen species/mitochondria theory, the DNA/genetic theory, and the telomerase theory, ${ }^{1,2}$ although none of these theories completely accounts for aging. Reactive oxygen species (ROS) are candidates for aging factors. ROS are normal by-products of cellular metabolism. Under basal conditions, $3-5 \%$ of the total oxygen consumed by mitochondria is converted to ROS. ${ }^{3,4}$ Therefore, mitochondria are thought to be exposed to ROS during a lifetime. The accumulation of ROS damage during aging oxidizes lipids, consequently modifying membrane lipid composition and reducing its fluidity. Finally, ROS increase mitochondrial membrane rigidity and reduce efficiency of mitochondrial oxidative phosphorylation, resulting in cell apoptosis. ${ }^{5,6}$ Thus, regulation of ROS may be a target for anti-aging therapy or treatment of age-associated diseases. Catalase is an anti-oxidant enzyme and catalyzes the reduction of hydrogen peroxide $\left(\mathrm{H}_{2} \mathrm{O}_{2}\right)$ before $\mathrm{H}_{2} \mathrm{O}_{2}$ is converted to hydroxyl radicals, which are highly aggressive. Schriner et al. reported that overexpression of catalase in mitochondria leads to a reduction of oxidative stress and an extension of lifespan. ${ }^{7}$ In addition, age-associated diseases including cardiovascular diseases and diabetes have been reported to be improved by antioxidant agents. ${ }^{8,9}$

The age-associated changes of human voices include harshness, reduced loudness, increased breathiness, and unsteadiness, ${ }^{10}$ which can lead to an impairment in the quality of life. These age-associated changes result from several factors including the loss of respiratory function, laryngeal muscle atrophy, neurological changes, and microstructural changes of the vocal fold mucosa. Previous studies investigated age-associated histological changes in the lamina propria (LP) of the vocal folds. It was 
reported that elastic fibers and hyaluronic acid (HA) were reduced and, conversely, collagenous fibers were increased with aging. ${ }^{11-15}$ Chen and Thibeault investigated gene expression of human vocal fold fibroblasts from various aged donors and showed that gene expression levels for collagen I, collagen VI, procollagen I and elastin decreased with increasing age of the donor. ${ }^{16}$ These age-associated changes of gene expression may result in age-associated histological changes of vocal folds.

Astaxanthin is a xanthophyll carotenoid and occurs naturally in living organisms, particularly in the marine environment. ${ }^{17,} 18$ Astaxanthin has a strong antioxidant effect as a potent quencher of singlet oxygen and many studies have shown favorable effects of astaxanthin on the regulation of oxidative stress..$^{8,18-20}$ For example, Gross et al. demonstrated that the administration of astaxanthin decreased myocardial infarct size through reducing ischemia-reperfusion injury caused by ROS..$^{20}$ Hama et al. reported that topical administration of astaxanthin to the skin prevented damage induced through oxidative stress by ultraviolet irradiation. ${ }^{21}$ Regarding the vocal folds, we demonstrated previously that astaxanthin treatment significantly reduced oxidative stress in the LP during the early phases of vocal fold wound healing and significantly reduced tissue contraction with favorable deposition of HA in the LP. ${ }^{22}$

Considering the ROS aging theory, we hypothesized that a reduction of oxidative stress in the cells of the vocal fold, such as fibroblasts, may lead to the attenuation of down-regulation of gene expression involved in ECM organization and prevent age-associated changes of vocal folds. To address this hypothesis, in the current study, we investigated the effect of astaxanthin on age-associated histological changes of vocal folds. After feeding rats on a diet containing astaxanthin for 12 months, we examined the histology and immunohistochemistry of 4-hydroxy-2-nonenal (4-HNE), an 
oxidative stress marker. In addition, to investigate the effect of astaxanthin on LP cells, we performed quantitative PCR of genes involved in regulating the organization of the LP. 


\section{Materials and methods}

\section{Animals and drug treatment}

The astaxanthin material, composed of $5.0 \%$ or $2.0 \%(\mathrm{w} / \mathrm{w})$ of astaxanthin was extracted from Haematococcus pluvialis, which was supplied by Fuji Chemical Industry Co. Ltd. (Toyama, Japan).

Thirty Sprague-Dawley rats were used in this study. Ten thirteen-month-old rats were used to investigate the transmission into vocal fold mucosa of astaxanthin, which was administered orally. After $100 \mathrm{mg} / \mathrm{day} / \mathrm{kg}$ astaxanthin was administered by oral gavage daily for seven days, rats were humanely euthanized with an intracardiac injection of sodium pentobarbital $(200 \mathrm{mg} / \mathrm{kg})$ after inhalative sedation with diethyl ether. And then whole larynges were harvested for high-performance liquid chromatography (HPLC).

Twenty six-month-old male rats were used to investigate the age-associated changes of vocal fold. Ten rats were fed a normal powder diet supplemented with $0.01 \%$ (w/w) astaxanthin (aged Ast-treated group), while the other ten rats were fed a normal powder diet without astaxanthin (aged sham-treated group). The astaxanthin material, composed of $2.0 \%(\mathrm{w} / \mathrm{w})$ astaxanthin, was mixed with the powder diet. After 12 months of feeding, the rats were humanely euthanized as described above. Whole larynges were harvested from each animal and then the larynges were split into the left and right sides. The left sides of larynges were used for histological and immunohistochemical examination, while the right sides were used for real-time PCR.

Since Sprague-Dawley rats live to be approximately 24 months, eighteen-month-old rats were selected in the current study to represent aged animals. Thirteen-week-old rats were used as a young control group (young group). 
All experimental protocols were approved by the Animal Research Committee of the Kyoto University Graduate School of Medicine. Animal care was provided by the Institute of Laboratory Animals of Kyoto University.

\section{High-performance liquid chromatography (HPLC)}

In order to examine transmission of astaxanthin into the vocal fold, the whole larynges of ten rats were harvested; the epithelium and lamina propria of the vocal fold were dissected away from the larynx under the microscope. The dissected specimens were frozen quickly in liquid nitrogen and stored at $-80^{\circ} \mathrm{C}$ until use. Since the vocal folds of rats are small, twenty vocal fold mucosa from the ten rats were put all together into one sample. Astaxanthin was extracted from the vocal fold mucosa with $2 \mathrm{ml}$ acetone at room temperature. The extract solution was concentrated by evaporating. The residue was dissolved in $50 \mu \mathrm{l}$ of acetone/hexane (2:8) solution and subjected to HPLC analysis. HPLC was performed on a Hitachi (Tokyo, Japan) L-6200 intelligent pump with a Hitachi L-4250 UV-VIS detector set at $470 \mathrm{~nm}$ absorbance. Astaxanthin was detected by HPLC on a Cosmosil 5SL-II $250 \times 4.6$ mm i.d., column (Nacalai Tesque, Kyoto, Japan) with acetone/hexane as a solvent at a flow rate of $1.0 \mathrm{ml} / \mathrm{min}$ at room temperature. Standard astaxanthin was provided by DSM Nutrition Japan (Tokyo, Japan).

\section{Immunohistochemical and histological examinations}

Age-related histological changes of the vocal fold were examined in the rats that were fed with or without astaxanthin. The harvested left sides of the larynges were immersed in $4 \%$ paraformaldehyde for 24 hours then in $30 \%$ sucrose for 24 hours. The tissues were then soaked in embedding medium (Optimum Cutting Temperature 
Compound; Tissue-Tek Inc., Kyoto, Japan) and frozen quickly with liquid nitrogen. Ten $\mu$ m-thick cryostat sections (Leica, CM 1850 Kryostat; Leica Instruments GmbH, Nussloch, Germany) of larynges were made vertical to the glottis (coronal sections), air dried, and stored at $-80^{\circ} \mathrm{C}$ until use.

For immunohistochemical examination, specimens were incubated overnight at $4^{\circ} \mathrm{C}$ with a rabbit anti-4-HNE antibody (Alpha Diagnostic International Co., San Antonio, TX, USA, 1:500) after blocking. Samples were washed, then incubated overnight at $4^{\circ} \mathrm{C}$ with Alexa-Fluor 488 goat anti-rabbit IgG (Invitrogen, France, 1:500). 4', 6'-diamidino-2-phenylindole dihydrochloride (DAPI) was used to stain cell nuclei in each specimen. Omission of the primary antibody served as a negative control. Images were captured with a Biorevo BZ-9000 microscope (Keyence Co., Osaka, Japan). Immunohistochemical staining of all the samples occurred simultaneously and all images were captured at 10X magnification with the same exposure and shutter speed. For histological examination, Alcian blue staining and a hyaluronidase digestion technique were performed to identify HA. In addition, Elastica van Gieson staining was performed to identify collagen. Images were captured at $10 \mathrm{X}$ magnification with a Biorevo BZ-9000 microscope.

Biorevo BZ-H1C and BZ-H1M software (Keyence Co.) was used to measure the 4-HNE-immunopositive areas in immunohistochemistry and blue-stained areas in Alcian blue staining. This software automatically measures the area of a designated color in a section. The threshold of 4-HNE-immunopositive or blue-stained color was determined by the examiner and saved by the computer. Under the same color criteria, the 4-HNE-immunopositive and blue-stained areas were measured in the entire lamina propria of three different regions in the vocal fold: anterior, middle, and 
posterior portions. These areas were then totaled (S1) for each vocal fold. In addition, the areas of the lamina propria were measured in all the regions and totaled (S2) for each vocal fold. The ratio of 4-HNE-immunopositive area, or blue-stained area, was determined by dividing S1 by S2 for each vocal fold. The ratio of HA-stained area in the LP was determined by subtracting the ratio of the blue-stained area of the section with hyaluronidase digestion from that of an adjacent section without digestion. These assessments were performed in a blinded fashion in which the examiners were not informed of the experimental group to which each section belonged.

\section{Real-time PCR}

In order to examine gene expression in the vocal folds, the harvested right sides of larynges were immediately immersed in RNAlater (QIAGEN, Valencia, CA, USA), incubated overnight, and then the epithelium and LP of the vocal fold were dissected away from the larynx under the microscope. The dissected specimens were frozen quickly in liquid nitrogen and stored at $-80^{\circ} \mathrm{C}$ until use. The RNeasy Mini Kit (QIAGEN) was used to isolate total RNA following the manufacturer's protocol. The quantity and quality of the RNA were evaluated by measuring the A260/280 ratio of the RNA samples. Reverse transcription was performed using a High Capacity cDNA Reverse Transcription Kit (Applied Biosystems, Foster City, CA, USA) using the manufacturer's recommended reaction protocol. Reactions were performed with a Veriti Thermal Cycler (Applied Biosystems) using the following parameters: $25^{\circ} \mathrm{C}$ for 10 minutes, $37^{\circ} \mathrm{C}$ for 120 minutes, $85^{\circ} \mathrm{C}$ for 5 minutes, and $4^{\circ} \mathrm{C}$ for 5 minutes.

We examined the mRNA expression of hyaluronic acid synthase (HAS)-1, HAS-2, and HAS-3, procollagen types I and III, matrix metallopeptidase (MMP)-1a, MMP-8, basic fibroblast growth factor (bFGF), hepatocyte growth factor (HGF), and 
beta 2-microglobulin (as a endogenous control). Rat-specific primers were used. The primers were synthesized by Hokkaido System Science Co, Japan and are displayed in Table 1. Real-time PCR was performed in a final volume of $20 \mu \mathrm{L}$ according to the manufacturer's protocol. The reaction mix was comprised of template cDNA, $10 \mu \mathrm{L}$ of Power SYBR Green Master Mix (Applied Biosystems), $0.25 \mu \mathrm{mol} / \mathrm{L}$ final concentration of each primer, and ribonuclease-free water. The amplification was performed under the following conditions: $95^{\circ} \mathrm{C}$ for 10 minutes, and 40 cycles of denaturing at $95^{\circ} \mathrm{C}$ for 15 seconds and annealing at $60^{\circ} \mathrm{C}$ for 1 minute. Fluorescence was detected with StepOnePlus (Applied Biosystems). Standard curves were used to determine the relative ratio of gene expression for each gene. Target gene ratios were normalized using the endogenous control gene (beta 2-microglobulin).

\section{Statistical Analysis}

One-way factorial analysis of variance (ANOVA) followed by a Tukey-Kramer post hoc test was performed to investigate the differences of the mRNA expression of HAS-1, HAS-3, MMP-1a, MMP-8, and HGF between the three groups. A Kruskal-Wallis test followed by a Steel-Dwass post-hoc test was performed to investigate the difference of the expression areas of 4-HNE, HA ratio, and the mRNA expression of the other genes because the data failed tests for homogeneity of variance. Differences of $\mathrm{p}<0.05$ were regarded as statistically significant. 


\section{Results}

Transmission to vocal folds of astaxanthin

HPLC chromatogram of the extraction from the vocal fold mucosa showed the peak corresponding to astaxanthin (Fig. 1). Although this peak was so small that astaxanthin content could not be quantified, this suggests the existence of astaxanthin in the vocal fold mucosa.

Survival of aged rats

Seven of ten rats survived in the aged Ast-treated group, while five of ten survived in the aged sham-treated group.

Expression of 4-hydroxy-2-nonenal (4-HNE)

The expression of 4-HNE increased with aging in the LP of the vocal folds (Fig. 2). Image analysis showed a significant increase of 4-HNE-immunopositive areas in both aged groups as compared to the young group. There were no significant differences in the 4-HNE-immunopositive area between the aged sham-treated group and the Ast-treated group.

\section{Histological examination}

Alcian blue staining and a hyaluronidase digestion technique showed the deposition of HA (Fig. 3). Image analysis showed that the deposition of HA in the LP was significantly reduced in the aged sham-treated group compared to the young group. No significant differences were observed between the aged Ast-treated group and the young group $(\mathrm{p}=0.21)$, or between the two aged groups. Elastica van Gieson staining showed excessive deposition of disorganized collagen in the LP in both aged groups.

\section{Real-time PCR}

The mRNA expression ratios of several extracellular matrix genes were 
examined (Fig. 4). The two aged groups showed significantly down-regulated mRNA expression of HAS-1, HAS-2, HAS-3, procollagen types I and III, MMP-1a, and MMP-8 as compared to the young group. Regarding the expression of growth factor genes, there were no significant differences in the expression of bFGF $(p=0.36)$ or HGF $(p=0.30)$ between the aged Ast-treated group and the young group, although the aged sham-treated group showed significantly down-regulated expression of these genes as compared to the young group (Fig. 5). No significant differences were observed in the expressions of these genes between the two aged groups. 


\section{Discussion}

In the current study, the age-associated histological change of vocal folds was consistent with previous studies. ${ }^{13-15}$ The deposition of HA in the LP was significantly reduced in the aged sham-treated group compared to the young group. Excessive deposition of disorganized collagen was observed in the LP of the aged vocal folds. By immunohistochemical examination, the expression of 4-HNE increased significantly with aging, which suggests an age-associated accumulation of oxidative stress in the vocal folds. In addition, the mRNA expression level of genes involved in ECM organization of the LP decreased significantly with aging.

Unfortunately, the current preliminary study showed no significant differences in any of the examinations between the two aged groups. This study has some limitations. Possible reasons are a sampling size and bias. Although twenty rats (ten per each group) were used at the beginning of the study, just twelve rats survived and were analyzed at the endpoint of this study. And the animals that survived may be more tolerant of oxidative stress, which may lead to sampling bias. The small sample size and possible sampling bias may have affected the result analysis.

The other limitation of the current study is a lack of knowledge about what dose is required to be effective for the vocal fold. Astaxanthin administered orally can transmit into vocal fold mucosa, which is suggested by the current HPLC study. Our previous study showed that $100 \mathrm{mg} / \mathrm{kg} /$ day astaxanthin administered by oral gavage for six days could influence the vocal fold. ${ }^{22}$ However, it is difficult to continue the administration by oral gavage for a long time. Therefore, we decided to feed the rats a diet containing $0.01 \%(\mathrm{w} / \mathrm{w})$ astaxanthin by referencing a previous study. ${ }^{23}$ Nakano et al. fed seven-week-old rats a diet containing $0.01 \%(\mathrm{w} / \mathrm{w})$ astaxanthin and another 
antioxidant for 12 weeks and their examinations after feeding showed a significant reduction in the levels of various oxidative stress markers. However, the dose administered in the current study may not be enough. The administration of an increased amount of astaxanthin, or in combination with other antioxidants, might reveal a better outcome.

On the other hand, no significant difference was observed in the deposition of HA in the LP between the aged Ast-treated group and the young group, although the aged sham-treated group showed a significant reduction of HA compared to the young group. It is sure that the small sample size might influence the result analysis, but this suggests that the administration of astaxanthin might contribute to the prevention of an age-associated reduction of HA. Since HA is regarded as an essential molecule for ideal viscoelasticity of the vocal folds, the preservation of HA is thought to contribute to maintenance of favorable mucosal vibration. In addition, there were no significant differences in the mRNA expression of bFGF or HGF between the aged Ast-treated group and the young group, although the aged sham-treated group showed significantly down-regulated expression of these genes as compared to the young group. Growth factors such as bFGF and $\mathrm{HGF}$ have been reported to have the potential to improve age-associated change of vocal folds. ${ }^{24-27}$ Moreover, a clinical trial was performed in Japan using bFGF for aged vocal folds, which demonstrated bFGF was effective for aged vocal folds. ${ }^{28}$ In our previous study, ${ }^{22}$ astaxanthin administration significantly increased the expression of bFGF during wound healing. This previous study is consistent with the current results. Our current results might also suggest that continuous administration of astaxanthin leads to the prevention of age-associated changes in voices through preserving the deposition of HA in the LP and maintaining 
expression of growth factors such as HGF and bFGF. 


\section{Conclusions}

This preliminary study demonstrated that a continuous administration of astaxanthin might attenuate the age-associated reduction of the deposition of $\mathrm{HA}$ in the LP and expression of HGF and bFGF. This suggests that astaxanthin has the potential to prevent age-associated changes in human voices. 


\section{ACKNOWLEDGEMENTS}

This study was supported by the Advanced Research for Medical Products Mining Program of the National Institute of Biomedical Innovation. 


\section{References}

1. Bonawitz ND, Shadel GS. Rethinking the mitochondrial theory of aging: the role of mitochondrial gene expression in lifespan determination. Cell Cycle. 2007;6:1574-1578.

2. Gavrilov LA, Gavrilova NS. The quest for a general theory of aging and longevity. Sci Aging Knowledge Environ. 2003;2003:RE5.

3. Sen CK. The general case for redox control of wound repair. Wound Repair Regen. 2003;11:431-438.

4. Davies KJ, Quintanilha AT, Brooks GA, Packer L. Free radicals and tissue damage produced by exercise. Biochem Biophys Res Commun. 1982;107:1198-1205.

5. Camici GG, Shi Y, Cosentino F, Francia P, Luscher TF. Anti-aging medicine: molecular basis for endothelial cell-targeted strategies - a mini-review. Gerontology. 2011;57:101-108.

6. Paradies G, Petrosillo G, Paradies V, Ruggiero FM. Role of cardiolipin peroxidation and Ca2+ in mitochondrial dysfunction and disease. Cell Calcium. 2009;45:643-650.

7. Schriner SE, Linford NJ, Martin GM, Treuting P, Oqbum CE, Emond M, Coskun PE, Ladiges W, Wolf N, Van Remmen H, Wallace DC, Rabinovitch PS. Extension of murine life span by overexpression of catalase targeted to mitochondria. Science. 2005;308:1909-1911.

8. Fassett RG, Coombes JS. Astaxanthin: a potential therapeutic agent in cardiovascular disease. Mar Drugs. 2011;9:447-465.

9. Timmers S, Hesselink MK, Schrauwen P. Therapeutic potential of resveratrol in obesity and type 2 diabetes: new avenues for health benefits? Ann N Y Acad Sci. 2013;1290:83-89. 
10. Ramig LO, Gray S, Baker K, Corbin-Lewis K, Buder E, Luschei E, Coon H, Smith M. The aging voice: a review, treatment data and familial and genetic perspectives. Folia Phoniatr Logop. 2001;53:252-265.

11. Sato K, Hirano M. Age-related changes of elastic fibers in the superficial layer of the lamina propria of vocal folds. Ann Otol Rhinol Laryngol. 1997;106:44-48.

12. Hammond TH, Gray SD, Butler J, Zhou R, Hammond E. Age- and gender-related elastin distribution changes in human vocal folds. Otolaryngol Head Neck Surg. 1998;119:314-322.

13. Sato K, Hirano M, Nakashima T. Age-related changes of collagenous fibers in the human vocal fold mucosa. Ann Otol Rhinol Laryngol. 2002;111:15-20.

14. Madruga de Melo EC, Lemos M, Aragao Ximenes Filho J, Sennes LU, Nascimento Saldiva PH, Tsuji DH. Distribution of collagen in the lamina propria of the human vocal fold. Laryngoscope. 2003;113:2187-2191.

15. Ohno T, Hirano S, Rousseau B. Age-associated changes in the expression and deposition of vocal fold collagen and hyaluronan. Ann Otol Rhinol Laryngol. 2009;118:735-741.

16. Chen X, Thibeault SL. Characteristics of age-related changes in cultured human vocal fold fibroblasts. Laryngoscope. 2008;118:1700-1704.

17. Higuera-Ciapara I, Felix-Valenzuela L, Goycoolea FM. Astaxanthin: a review of its chemistry and applications. Crit Rev Food Sci Nutr. 2006;46:185-196.

18. Pashkow FJ, Watumull DG, Campbell CL. Astaxanthin: a novel potential treatment for oxidative stress and inflammation in cardiovascular disease. Am J Cardiol. 2008;101:58D-68D. 
19. Wolf AM, Asoh S, Hiranuma H, Ohsawa I, Iio K, Satou A, Ishikura M, Ohta S. Astaxanthin protects mitochondrial redox state and functional integrity against oxidative stress. J Nutr Biochem. 2010;21:381-389.

20. Gross GJ, Lockwood SF. Cardioprotection and myocardial salvage by a disodium disuccinate astaxanthin derivative (Cardax). Life Sci. 2004;75:215-224.

21. Hama S, Takahashi K, Inai Y, Shiota K, Sakamoto R, Yamada A, Thuchiya H, Kanamura K, Yamashita E, Kogure K. Protective effects of topical application of a poorly soluble antioxidant astaxanthin liposomal formulation on ultraviolet-induced skin damage. J Pharm Sci. 2012;101:2909-2916.

22. Mizuta M, Hirano S, Hiwatashi N, Tateya I, Kanemaru S, Nakamura T, Ito J. Effect of astaxanthin on vocal fold wound healing. Laryngoscope. 2013.

23. Nakano M, Orimo N, Katagiri N, Tsubata M, Takahashi J, Van Chuyen N. Inhibitory effect of astraxanthin combined with Flavangenol on oxidative stress biomarkers in streptozotocin-induced diabetic rats. Int J Vitam Nutr Res. 2008;78:175-182.

24. Hirano S, Bless DM, del Rio AM, Connor NP, Ford CN. Therapeutic potential of growth factors for aging voice. Laryngoscope. 2004;114:2161-2167.

25. Hirano S, Nagai H, Tateya I, Tateya T, Ford CN, Bless DM. Regeneration of aged vocal folds with basic fibroblast growth factor in a rat model: a preliminary report. Ann Otol Rhinol Laryngol. 2005;114:304-308.

26. Ohno T, Yoo MJ, Swanson ER, Hirano S, Ossoff RH, Rousseau B. Regenerative effects of basic fibroblast growth factor on extracellular matrix production in aged rat vocal folds. Ann Otol Rhinol Laryngol. 2009;118:559-564. 
27. Ohno T, Yoo MJ, Swanson ER, Hirano S, Ossoff RH, Rousseau B. Regeneration of aged rat vocal folds using hepatocyte growth factor therapy. Laryngoscope. 2009;119:1424-1430.

28. Hirano S, Tateya I, Kishimoto Y, Kanemaru S, Ito J. Clinical trial of regeneration of aged vocal folds with growth factor therapy. Laryngoscope. 2012;122:327-331. 


\section{Figures}

Figure 1

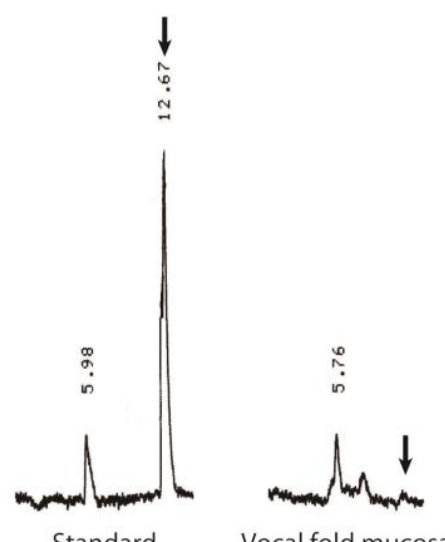

Standard

Vocal fold mucosa

HPLC chromatograms of the standard astaxanthin sample and the extraction from vocal fold mucosa. Arrow indicates the peaks corresponding to astaxanthin. 
Figure 2

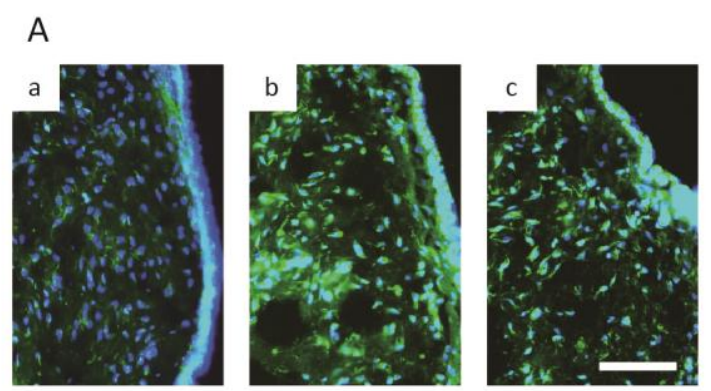

B

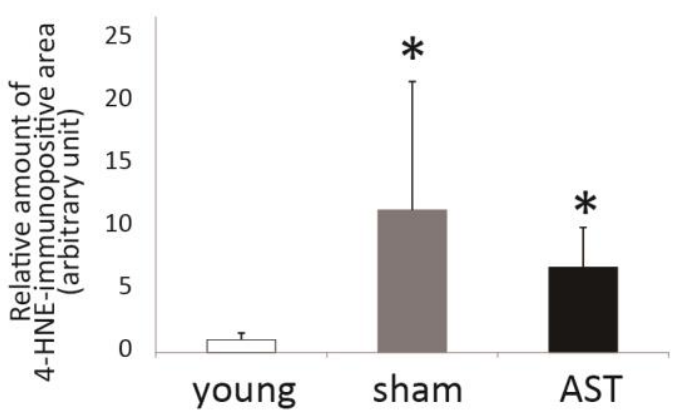

(A) Representative examples of immunohistochemistry for 4-hydroxy-2-nonenal (4-HNE) in the lamina propria of vocal folds in the young group (a), the aged sham-treated group (b), and the aged astaxanthin (Ast)-treated group (c). 4-HNE appears green, while nuclei appear blue. Scale bar: $100 \mu \mathrm{m}$. (B) The immunopositive area for 4-HNE was measured in the lamina propria and presented as a value relative to the young group. The ratio of the 4-HNE-immunopositive area significantly increased in the two aged groups compared to the young group. No significant differences were observed in the 4-HNE-positive area between the aged sham-treated group and the Ast-treated group. Bars represent the standard deviation. *: $\mathrm{p}<0.05$, compared to the young group. 
Figure 3

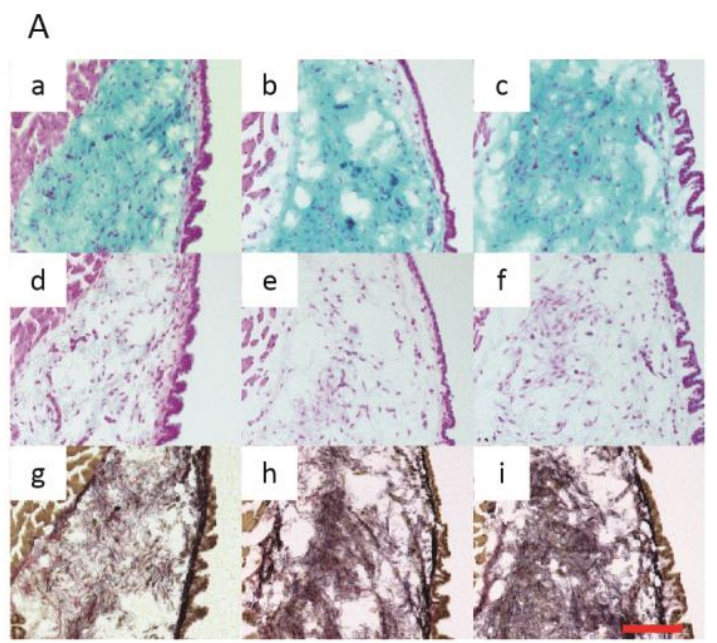

B

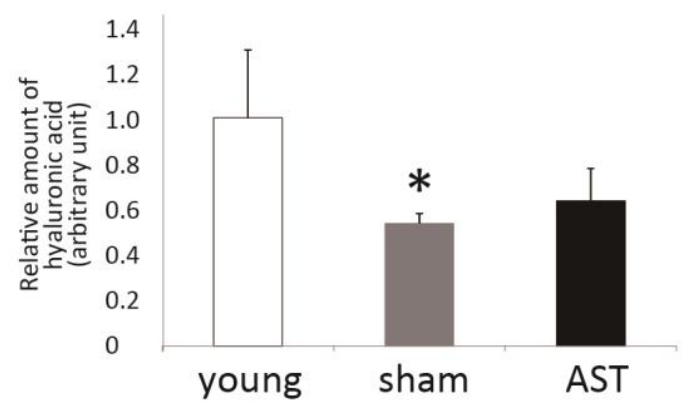

A) Representative Alcian blue staining without hyaluronidase digestion ( $a, b, c)$ and with hyaluronidase digestion (d, e, f), and Elastica van Gieson staining (g, h, i) of the young group (a, d, g), the aged sham-treated group (b, e, h), and the aged astaxanthin (Ast)-treated group (c, f, i). The young group had abundant deposition of hyaluronic acid (HA) in the lamina propria. The sham-treated aged group showed a decrease of HA deposition, which was attenuated in the aged Ast-treated group. Elastica van Gieson staining showed excessive deposition of disorganized collagen in the lamina propria in both aged groups. Scale bar: $100 \mu \mathrm{m}$.

B) The HA-stained area was measured and presented as a value relative to the young group. The deposition of HA in the LP was significantly reduced in the aged 
sham-treated group compared to the young group. No significant difference was observed between the aged Ast-treated group and the young group $(\mathrm{p}=0.21)$. Bars represent the standard deviation. ${ }^{*}: \mathrm{p}<0.05$, compared to the young group. 
Figure 4

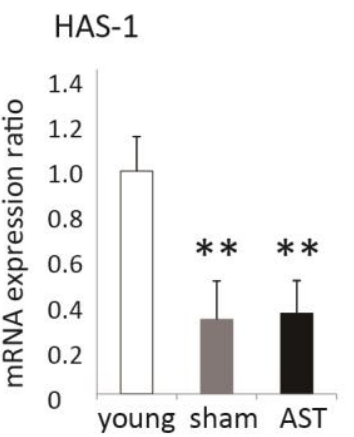

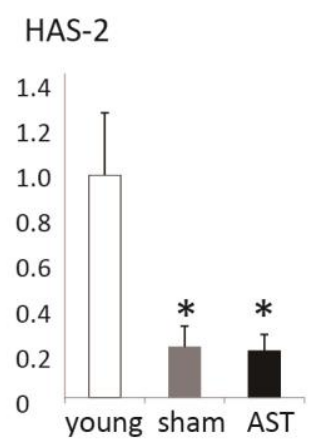

HAS-3

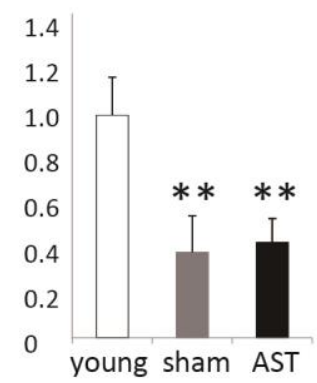

MMP-1a

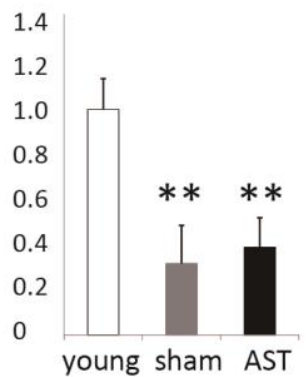

young sham AST

\section{MMP-8}

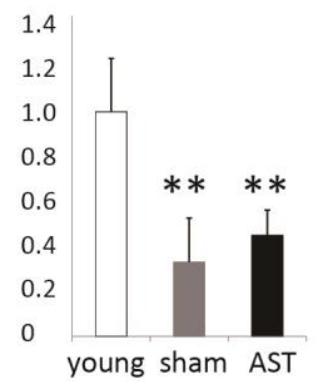

procollagen type I

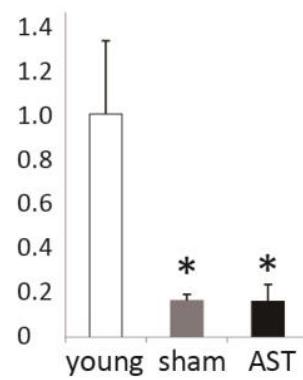

procollagen type III

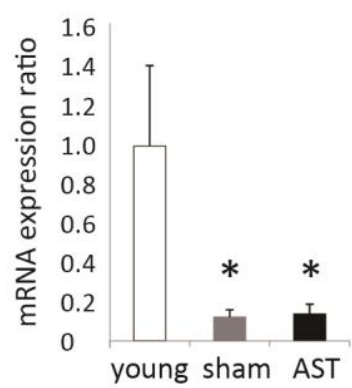

mRNA expression ratios of several genes involved in the organization of vocal folds in the young group, the aged sham-treated group, and the aged astaxanthin (Ast)-treated group (Ast). The two aged groups showed significantly down-regulated mRNA expression of hyaluronic acid synthase (HAS)-1, HAS-2, HAS-3, procollagen types I and III, matrix metallopeptidase (MMP)-1a, and MMP-8 as compared to the young group. No significant differences were observed in the expression of these genes between the two aged groups. Bars represent the standard deviation. ${ }^{* *}: \mathrm{p}<0.01{ }^{*}: \mathrm{p}<0.05$, compared to the young group. 
Figure 5

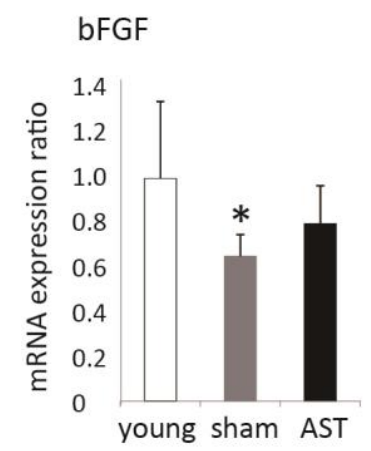

HGF

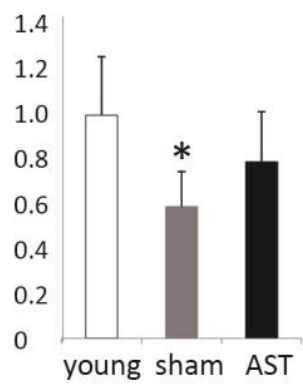

mRNA expression ratios of basic fibroblast growth factor (bFGF) and hepatocyte growth factor (HGF) in the young group, the aged sham-treated group, and the aged astaxanthin (Ast)-treated group. Although the aged sham-treated group showed a significantly down-regulated expression of bFGF and HGF as compared to the young group, there were no significant differences in the expression of these genes between the aged Ast-treated group and the young group. Bars represent the standard deviation. *: $\mathrm{p}<0.05$, compared to the young group. 
Table 1

Table 1: Primer Sequences

\begin{tabular}{|c|c|c|}
\hline Gene name & & Sequence \\
\hline \multirow{2}{*}{ Hyaluronic acid synthase 1} & Forward & 5'-TAGGTGCTGTTGGAGGAGATGTGA-3' \\
\hline & Reverse & 5'-AAGCTCGCTCCACATTGAAGGCTA-3' \\
\hline \multirow{2}{*}{ Hyaluronic acid synthase 2} & Forward & 5'-ACTGGGCAGAAGCGTGGATTATGT-3' \\
\hline & Reverse & 5'-AACACCTCCAACCATCGGGTCTTCTT-3' \\
\hline \multirow{2}{*}{ Hyaluronic acid synthase 3} & Forward & 5'-CCTCATCGCCACAGTCATACAA-3' \\
\hline & Reverse & 5'-CCACCAGCTGCACCGTTAGT-3' \\
\hline \multirow{2}{*}{ Procollagen type I } & Forward & 5'-AGGCATAAAGGGTCATCGTGGCTT-3' \\
\hline & Reverse & 5'-AGTCCATCTTTGCCAGGAGAACCA-3' \\
\hline \multirow{2}{*}{ Procollagen type III } & Forward & 5'-ATGAGCTTTGTGCAATGTGGGACC-3' \\
\hline & Reverse & 5'-ACTGACCAAGGTAGTTGCATCCCA-3' \\
\hline \multirow{2}{*}{ Matrix metallopeptidase 1a } & Forward & 5'-GAAACCCTGAGTGCTATGAG-3' \\
\hline & Reverse & 5'-TTTTGGCAAATATGGTGTGT-3' \\
\hline \multirow{2}{*}{ Matrix metallopeptidase 8} & Forward & 5'-CCATGGATCCAGGTTACCCCACT-3' \\
\hline & Reverse & 5'-TGTGGTCCACTGAAGAAGAGGAAGA-3' \\
\hline \multirow{2}{*}{ Basic fibroblast growth factor } & Forward & 5'-AAGAACGGCGGCTTCTTCCT-3' \\
\hline & Reverse & 5'-CCCTTGATGGACACAACTCC-3' \\
\hline \multirow{2}{*}{ Hepatocyte growth factor } & Forward & 5'-ACAAGGGCTTTCCATTCACT-3' \\
\hline & Reverse & 5'-CCAGTAGCATCGTTTTCTCG-3' \\
\hline \multirow{2}{*}{ Beta 2-microglobulin } & Forward & 5'-TCACACTGAATTCACACCCACCGA-3' \\
\hline & Reverse & 5'-TGATTACATGTCTCGGTCCCAGGT-3' \\
\hline
\end{tabular}

